\title{
Endomysial antibody in the diagnosis and management of coeliac disease
}

\author{
Martin W James, Brian B Scott
}

\begin{abstract}
This review determines the significance, usefulness, and application of the endomysial antibody test for coeliac disease in clinical practice.

(Postgrad Med F 2000;76:466-468)
\end{abstract}

Keywords: coeliac disease; endomysial antibody; screening

Coeliac disease is a disorder of the small intestine mediated by immunological processes initiated by exposure to dietary gluten in genetically predisposed individuals. Characteristic changes are recognised histologically and are reversible on the withdrawal of gluten from the diet. It is a common disorder, with recent population studies indicating a prevalence of one in 300 in Europe.

\section{Diagnosis}

Histology of small bowel biopsy specimens remains the "gold standard" for diagnosis. Features recognised include villus atrophy, crypt hyperplasia, degenerate surface epithelial cells, and an increase in intraepithelial lymphocytes. However, the severity of these changes is variable, ${ }^{2}$ with some untreated patients having a raised intraepithelial lymphocyte count alone. ${ }^{3}$ Coeliac disease is a curable condition which, left untreated, may result in nutritional deficiency and malignancy, ${ }^{4}$ particularly $\mathrm{T}$ cell lymphoma of the small bowel. Early diagnosis and treatment is therefore important. It is thought that the risk of malignancy is reduced by strict adherence to a gluten-free diet. ${ }^{4}$

It is becoming increasingly recognised that only a minority of patients with coeliac disease have classical symptoms such as failure to thrive in infancy, weight loss, and chronic diarrhoea. Many patients may be asymptomatic ("silent" coeliac disease), or may present with extraintestinal effects such as anaemia, dermatitis herpetiformis, osteomalacia, osteoporosis and infertility, or with an associated condition such as type I diabetes, ${ }^{5}$ obscure neurological disease, ${ }^{6}$ and primary biliary cirrhosis. ${ }^{78}$ Other patients may be detected as a result of family screening of an index patient.

Small bowel biopsy is always indicated when Gastroenterology, County Hospital, Lincoln LN2 5QY, UK

$\mathrm{M}$ W James

B B Scott

Correspondence to: Dr Scott

Submitted 20 September 1999

Accepted 16 November 1999 there is a high suspicion (that is a high pre-test probability) of coeliac disease It is reliable and technically straightforward by endoscopy, ${ }^{9}$ but relatively expensive, time consuming, and unpleasant for patients. It is thus not appropriate for testing large numbers when the index of suspicion is low. There is therefore a need for a less invasive screening test to select patients for biopsy.

\section{Serological tests}

Attempts to develop sensitive and specific serological tests to aid diagnosis started in 1958 when Berger described the antigliadin antibody (gliadin is the alcohol soluble fragment of gluten), ${ }^{10}$ which has been used clinically since the 1970 s. Further antibodies have been discovered including antireticulin, antijejunal, and endomysial antibody (EMA). ${ }^{11}$

The endomysium is the perivascular connective tissue which lines smooth muscle bundles, and which takes up silver stain. It has recently been suggested that the target antigen in endomysium is tissue transglutaminase. ${ }^{12}$ Tissue transglutaminase is a ubiquitous calcium dependent enzyme that crosslinks proteins. When it reacts with gliadin, neoepitopes are formed. It is thought that the immunological response to these neoepitopes may initiate the mucosal damage in coeliac disease.

\section{Endomysial antibody}

The EMA is also referred to as antiendomysial antibody. The commercially available tests for EMA detect IgA class autoantibody directed against the endomysium in monkey oesophagus by indirect immunofluorescence, as first described by Chorzelski et al in $1983 .{ }^{11}$ More recent work using human umbilical cord tissue as a substrate has shown improved sensitivity and correlation with villus atrophy, and has overcome the ethical issue of using samples from endangered species. ${ }^{13} 14$

The technique of indirect immunofluorescence for IgA EMA is both subjective and more labour intensive than the ELISA tests which are used for IgA and IgG antigliadin antibody. However, it has been consistently demonstrated that EMA has superior sensitivity and specificity than assays for antigliadin and antireticulin antibodies. Since selective IgA deficiency is much more common in coeliac disease $(2 \%-3 \%$, compared with $0.2 \%$ in the general population), a simultaneous immunoglobulin assay should be performed. In patients with such deficiency, the IgA EMA test is rendered useless, and a small bowel biopsy is necessary. ${ }^{15} 16$

PERFORMANCE OF THE EMA TEST

Review of all published peer reviewed studies from 1985 to 1999 shows the sensitivity varies from $74 \%$ to $100 \%$ and the specificity from $64 \%$ to $100 \%$. Critical assessment of the pooled data (excluding one study which had several defects and a specificity well below any other study ${ }^{17}$ ), which consists of 2006 untreated coeliacs and 4107 apparent noncoeliacs, shows that the test has a sensitivity of $94 \%$ and a specificity of $99 \% .^{13} 1417-35$ 
It should be understood that the post-test probability of coeliac disease given a positive or negative test is critically dependent on the prevalence of coeliac disease in the population being studied. The lower the prevalence, the lower the post-test probability. Thus, if the prevalence is low a negative test virtually excludes the diagnosis. However, if the prevalence is high then the probability of coeliac disease, even with a negative test, is sufficiently high to warrant biopsy. Using the EMA test in this situation is inappropriate.

LATENT COELIAC DISEASE?

One striking feature is the relatively few false positive EMA tests (51/4107). Furthermore, it could be that the false positive rate is even lower. In one of the studies, five of the 39 who had a positive EMA test and normal small bowel histology consented to rebiopsy within 16 months. ${ }^{21}$ All had a flat mucosa at second biopsy. Another study reported 10 patients with normal mucosal architecture and positive EMA tests. ${ }^{36}$ They all improved symptomatically on a gluten-free diet and the EMA test became negative. Furthermore, there was evidence of intestinal immune activation (for example, increased expression of the intracellular adhesion molecule ICAM-1, and the presence of lamina propria mononuclear cells bearing CD25 and CD80). These findings support the concept of latent coeliac disease. Another possible explanation for these findings is that abnormal mucosal architecture may have been missed by sampling error in a condition known to have patchiness of mucosal abnormality. ${ }^{37}$ In either case, it could be that EMA positivity is a better marker of coeliac disease than histology!

This evidence suggests that in EMA positive patients with normal histology, the biopsies should be repeated, taking multiple specimens and examining them in great detail including intraepithelial lymphocyte counting (which was abnormal in $40 \%$ of the "normal" histology patients in the second study $\left.{ }^{36}\right)$. Even if still normal, a trial of gluten withdrawal should be considered if the patient is symptomatic.

The authors are aware of further examples in their district of patients with a positive EMA test, normal routine small bowel histology, and symptoms that responded to a gluten-free diet. This concept does not fit easily with the standard teaching or criteria for the diagnosis of coeliac disease that stipulates mucosal changes that regress with gluten withdrawal, and/or reappear with gluten challenge.

ENDOMYSIAL ANTIBODY AS A MEASURE OF MUCOSAL RESPONSE AND DIETARY COMPLIANCE? A frequent observation is that EMA becomes negative on withdrawal of gluten from the diet in patients with proved coeliac disease. The question then arises whether EMA could be used to assess patients' compliance with the diet, and if there is concordance between EMA positivity and mucosal architecture. SategnaGuidetti et al proposed that EMA titre was indirectly related to mucosal recovery. ${ }^{35}$ However, more recent work by the same authors
Endomysial antibody test in coeliac disease

- Specificity $99 \%$

- Sensitivity $94 \%$

- Cannot be used in IgA deficiency

- Useful for screening when pre-test probability low (for example, in type I diabetes)

- Unnecessary when pre-test probability high — go straight to small bowel biopsy

- Positive test may indicate latent coeliac disease when biopsy normal (?keep under review, repeat multiple biopsies, consider gluten-free diet if symptoms)

- Not reliable in monitoring response to treatment

concluded that the kinetics of EMA and its relationship to mucosal recovery after gluten withdrawal have yet to be determined. EMA positivity was a predictor of persistent villus atrophy, but a negative test was not a reliable indicator of mucosal recovery. EMA positivity in patients on a gluten-free diet varied from 0 to $68 \%,{ }^{19}$ but this may reflect the unreliability of dietary inquiry. EMA negativity may reflect the absence of gluten in the diet in those who were initially positive, but is not a predictor of mucosal outcome. Biopsy remains the best tool in this respect. ${ }^{38} 39$

\section{Conclusion}

The EMA test has high sensitivity and specificity (except in IgA deficiency). It is very useful for screening those in whom coeliac disease is suspected but in whom the probability of the disease is not high - for example less than 20\% (such as in type I diabetes). Where the suspicion is higher (such as dermatitis herpetiformis or a young person with diarrhoea, weight loss, and anaemia where the probability is around $80 \%$ ) then a small bowel biopsy should be done without EMA testing. If an EMA test is positive it should be followed by small bowel biopsy. If the biospy is normal it would be prudent to keep the patient under review and repeat the biopsy-taking multiple samples. If still normal and yet symptomatic a gluten-free diet could be tried, although objective evidence of response may be difficult. Repeating the EMA test in coeliacs on a gluten-free diet is not useful. Now the antigen in endomysium is known (tissue transglutaminase), antibody tests to transglutaminase may prove to be even more useful than EMA. However, the current tests need more refinement and are not yet recommended for routine clinical practice.

1 Catassi C, Ratch I-M, Fabiani E, et al. Coeliac disease in the year 2000: exploring the iceberg. Lancet 1994;343:200-3.

2 Scott BB, Losowsky MS. Coeliac disease with mild mucosal abnormalities: a report of four patients. Postgrad Med $\mathcal{F}$

3 Ferguson A, Murray D. Quantitation of intraepithelial lymphocytes in human jejunum. Gut 1971;12:988.

4 Hocytes in human jejunum. Gut 1971;12:988. liac disease- effect of a gluten-free diet. Gut 1989;30:333-8. 
5 Cronin CC, Shanahan F. Insulin-dependent diabetes mellitus and coeliac disease. Lancet 1997;349:1096-7.

6 Hadjivassilou M, Gibson A, Davies-Jones GAB, et al. Does cryptic gluten sensitivity play a part in neurological illness. Lancet 1996:347:369-71.

7 Kingham JG, Parker DR. The association between primary biliary cirrhosis and coeliac disease: a study of relative prevalences. Gut 1998;42:120-2.

8 Sorensen HT, Thulstrup AM, Blomqvist P, et al. Risk of primary biliary cirrhosis in patients with coeliac disease: Danish and Swedish cohort study. Gut 1999;44:736-8.

9 Scott BB, Jenkins D. Endoscopic small intestinal biopsy. Gastrointest Endosc 1981;27:162-7.

10 Berger E. Zur allergischen Pathogenese der Coliakie. Bibliothecs Paediatrica 1958;67(suppl 1):1-55.

11 Chorzelski TP, Sulej T, Tchorzewski H, et al. IgA class endomysium antibodies in dermatitis herpetiformis and coeliac disease. Ann N Y Acad Sci 1983;420:325-34.

12 Dieterich W, Ehnis T, Bauer M, et al. Identification of tissue transglutaminase as the autoantigen of celiac disease. Nat Med 1997;3:797-801.

13 Ladinser B, Rossipal E, Pittschieler K. Endomysium antibodies in coeliac disease: an improved method. Gut antibodies in coel

14 Corraccio A, Ccavataio F, Iacono G, et al. Ig A antiendomysial antibodies on the umbilical cord in diagnosing celiac disease. Scand f Gastroenterol 1996;31:759-63.

15 Cataldo F, Marino V, Ventura A, et al, and the Italian Society of Paediatric Gastroenterology and Hepatology (SIGEP) and "Club del Tenue" working groups on coeliac disease. Gut 1998;42:362-5.

16 Rittmeyer C, Rhooads JM. IgA deficiency causes falsenegative endomysial antibody results in coeliac disease. $\mathscr{f}$ Pediatr Gastroenterol Nutr 1996;23:504-6.

17 Atkinson K, Tokmakajian S, Watson W, et al. Evaluation of the endomysial antibody for coeliac disease: operating properties and associated cost implications in clinical practice. Can 7 Gastroenterol 1997;11:673-7.

18 Volta U, Molinaro N, Fusconi M, et al. IgA antiendomysial antibody test. A step forward in celiac disease screening. Dig antibody test. A step fo

19 Ferreira M, Lloyd Davies S, Butler M, et al. Endomysial antibody: is it the best screening test for coeliac disease? Gut 1992;33:1633-7.

20 Sategna Guidetti C, Grosso S, Bruno M, et al. Comparison of serum anti-gliadin, anti-endomysium, and anti-jejunum antibodies in adult coeliac sprue. F Clin Gastroenterol 1995 20:17-21

21 Cataldo F, Ventura A, Lazzari R, et al. Antiendomysium antibodies and coeliac disease: solved and unsolved questions. An Italian multicentre study. Acta Paediatr 1995; 84:1125-31.

22 Ascher H, Hahn-Zoric M, Hanson LA, et al. Value of serological markers for clinical diagnosis and population studies of coeliac disease. Scand 7 Gastroenterol 1996;31:61-7.

23 Volgelsang H, Genser D, Wyatt J, et al. Screening for coelica disease: a prospective study on the value of noninvasive disease: a prospective study on the value
tests. Am f Gastroenterol 1995;90:394-7.
24 Kapuscinska A, Zalewski T, Chorzelski TP, et al. Disease specificity and dynamics of changes in IgA class antiendomysial antibodies in coeliac disease. $f$ Pediatr Gastroenterol Nutr 1987;6:529-34.

25 Rossi TM, Kumar V, Lerner A, et al. Relationship of endomysial antibodies to jejunal mucosal pathology: specificity towards both symptomatic and asymptomatic coeliacs. $\mathcal{F}$ Pediatr Gastroenterol Nutr 1988;7:858-63.

26 Kumar V, Lerner A, Valeski JE, et al. Endomysial antibodies in the diagnosis of coeliac disease and the effect of gluten on antibody titres. Immunol Invest 1989;18:533-44.

27 Hallstrom O. Comparison of IgA-class reticulin and endomysium antibodies in coeliac disease and dermatitis herpetiformis. Gut 1989;30:1225-32.

28 Burgin-Wolff A, Gaze H, Hadziselimovic F, et al. Antigliadin and antiendomysium antibody determination for coeliac disease. Arch Dis Child 1991;66:941-7.

29 McMillan SA, Haughton DJ, Biggart JD, et al. Predictive value for coeliac disease of antibodies to gliadin, endomysium, and jejunum in patients attending for jejunal biopsy. BMF 1991;303:1163-5.

30 Mascart-Lemone F, Lambrechts A. Serolgy of coeliac disease:early diagnosis and therapeutic impact. Acta Gastroenterol Belg 1995;58:388-96.

31 Valdimarsson T, Franzen L, Grodzinsky E, et al. Is small bowel biopsy necessary in adults with suspected coeliac disease and IgA anti-endomysium antibodies? Dig Dis Sci 1996;41:83-7.

32 Lerner A, Blank M, Lahat N, et al. Increased prevalence of autoantibodies in coeliac disease. Dig Dis Sci 1998;43:723-

33 Sulkanen S, Halttunen $\mathrm{T}$, Laurila $\mathrm{K}$, et al. Tissue transglutaminase autoantibody enzyme-linked immunosorbent assay in detecting coelisc disease. Gastroenterology 1998;115:1322-8.

34 Feighery C, Weir DG, Whelan A, et al. Diagnosis of glutensenstive enteropathy: is exclusive reliance on histology appropriate? Eur f Gastroenterol. Hepatol 1998;10:919-25.

35 Sategna-Guidetti C, Pulitano R, Grosso S, et al. Serum IgA antiendomysium antibody titres as a marker of intestinal involvement and diet compliance in adult celiac sprue. $\mathcal{F}$ Clin Gastroenterol 1993;17:123-7.

36 Picarelli A, Maiuri L, Mazzilli MCP, et al. Gluten-sensitive disease with mild enteropathy. Gastroenterology 1996;111: 608-16.

37 Scott BB, Losowsky ML. Patchiness and duodeno-jejunal variation of the mucosal abnormality in coeliac disease and dermatitis herpetiformis. Gut 1976;17:984-92.

38 Sategna-Guidetti C, Grosso S, Bruno M, et al. Reliability of immunological markers of coeliac sprue in the assessment of mucosal recovery after gluten withdrawal. f Clin Gastroenterol 1996;23:101-4.

39 Troncone R, Mater M, Ispagnuolo F, et al. Endomysial antibodies an unreliable marker for slight dietary transgression in adolescents with coeliac disease. 7 Pediatr Gastroenterol Nutr 1995;21:69-72. 